Ferrata Storti Foundation

\title{
The process of somatic hypermutation increases polyreactivity for central nervous system antigens in primary central nervous system lymphoma
}

Haematologica 2021

Volume 106(3):708-717

\section{Correspondence:}

MARTINA DECKERT

martina.deckert@uni-koeln.de

Received: November 8, 2019.

Accepted: March 18, 2020.

Pre-published: March 19, 2020.

https://doi.org/10.3324/haematol.2019.242701

(C)2021 Ferrata Storti Foundation

Material published in Haematologica is covered by copyright. All rights are reserved to the Ferrata Storti Foundation. Use of published material is allowed under the following terms and conditions:

https://creativecommons.org/licenses/by-nc/4.0/legalcode. Copies of published material are allowed for personal or internal use. Sharing published material for non-commercial purposes is subject to the following conditions:

https://creativecommons.org/licenses/by-nc/4.0/legalcode, sect. 3. Reproducing and sharing published material for commercial purposes is not allowed without permission in writing from the publisher.

\section{Manuel Montesinos-Rongen, ${ }^{1}$ Monica Terrao, ${ }^{1}$ Caroline May, ${ }^{2}$ Katrin Marcus, ${ }^{2}$ Ingmar Blümcke, ${ }^{3}$ Martin Hellmich, ${ }^{4}$ Ralf Küppers, ${ }^{5}$ Anna Brunn ${ }^{1}$ and Martina Deckert ${ }^{1}$}

${ }^{1}$ Institute of Neuropathology, Faculty of Medicine and University Hospital Cologne, University of Cologne, Cologne; ${ }^{2}$ Medizinisches Proteom-Center, Ruhr-University Bochum, Bochum; ${ }^{3}$ Department of Neuropathology, University Hospital Erlangen, Erlangen, Germany; ${ }^{4}$ Institute of Medical Statistics and Computational Biology (IMSB), Faculty of Medicine and University Hospital Cologne, University of Cologne, Cologne and Institute of Cell Biology (Cancer Research), University of Duisburg-Essen, Medical School, Essen, Germany, and German Cancer Consortium (DKTK), Essen/Düsseldorf, Germany

\section{ABSTRACT}

The immunoglobulin (Ig) heavy and light chain variable gene mutational pattern of the B-cell receptor (BCR) in primary central nervous system lymphoma (PCNSL) cells suggests antigenic selection to drive pathogenesis and confinement to the central nervous system (CNS). This hypothesis is supported by the observation that the tumor B-cell receptor (tBCR) of PCNSL is polyreactive and may be stimulated by CNS proteins. To obtain further insight into the role of the germinal center (GC) reaction on BCR reactivity, we constructed recombinant antibodies (recAb) with Ig heavy and light chain sequences of the corresponding naïve $\mathrm{BCR}$ ( $\mathrm{BCCR}$ ) by reverting tBCR somatic mutations in ten PCNSL. Analysis of $\mathrm{nBCR}$-derived recAb reactivity by a protein microarray and immunoprecipitation demonstrated auto- and polyreactivity in all cases. Self/polyreactivity was not lost during the GC reaction; surprisingly, tBCR significantly increased self-/polyreactivity. In addition to proteins recognized by both the $\mathrm{nBCR}$ and $\mathrm{tBCR}, \mathrm{tBCR}$ gained self-/polyreactivity particularly for proteins expressed in the CNS including proteins of oligodendrocytes/myelin, the S100 protein family, and splicing factors. Thus, in PCNSL pathogenesis, a faulty GC reaction may increase self-/polyreactivity, hereby facilitating BCR signaling via multiple CNS antigens, and may ultimately foster tumor cell survival in the CNS.

\section{Introduction}

Primary lymphoma of the central nervous system (PCNSL) is a distinct diffuse large B-cell lymphoma (DLBCL) entity confined to the central nervous system (CNS). ${ }^{1}$

PCNSL tumor cells carry somatically mutated rearranged immunoglobulin (Ig) heavy and light chain variable genes, ${ }^{2,3}$ revealing a germinal center (GC) experience of the lymphoma cells. ${ }^{4}$ A prerequisite for the GC reaction is a unique micromilieu in which follicular dendritic cells present cognate antigen to $\mathrm{B}$ cells and helper $\mathrm{T}$ cells mediate B-cell selection, aiming to increase B-cell receptor (BCR) affinity for the antigen. Considering confinement to the CNS, the observation of ongoing somatic hypermutation (SHM), ${ }^{2}$ a GC B-cell-specific process, raises the intriguing question as to the impact of the target organ, particularly of CNS antigens, on this organ-specific DLBCL entity. While T-helper cells and antigen presenting cells are present in the PCNSL-infiltrated CNS, their in vivo characteristics and function have not yet been elucidated. Thus, it is still unknown whether a GC reaction indeed occurs in the CNS. Despite the observation of follicle-like structures in the leptomeninges, but not the brain parenchyma, in some patients with late multiple scle- 
rosis phases, ${ }^{5}$ formal proof of GC and a GC reaction in the brain is still lacking. In addition to a lack of classical, fully functional GC in the brain, the CNS microenvironment may also, at least in part, be responsible for the absence of Ig class switch in PCNSL due to a lack of signals in the GC required for class switching. The recent demonstration that SHM and class switch recombination occur independently and are topographically distinct is in line with this notion. ${ }^{6}$

So far, studies of PCNSL aiming at the identification of foreign antigens that might trigger the tumor $\mathrm{B}$ cells have been inconclusive; in particular, viruses able to persist in the CNS, e.g., HHV6, HHV8, and SV40 were excluded. ${ }^{7-9}$ Taking advantage of the fact that the tumor cells of PCNSL express a functional BCR, we previously reconstructed the tumor cell BCR (tBCR) as recombinant antibodies (recAb) in 23 PCNSL to identify their antigen recognition pattern on a large-scale protein microarray. This approach aimed at the identification of proteins that were expressed in the $\mathrm{CNS}$ and on the cell surface, thus enabling their recognition as antigen by the BCR; it revealed that the tumor cells are polyreactive including reactivity with proteins physiologically expressed in the CNS. Neuronally expressed GRINL1A, centaurin, and BAIAP2 were recognized by tBCR. ${ }^{10}$ The majority of $\mathrm{tBCR}$ recognized galectin-3, upregulated on microglia/macrophages, astrocytes, and endothelial cells upon CNS invasion by PCNSL. ${ }^{10}$ Thus, proteins differentially expressed by resident CNS cell populations may trigger the $\mathrm{tBCR}$ and support PCNSL survival in the CNS.

These data prompted us to revert somatic mutations of the $\mathrm{tBCR}$ to their preimmune sequence, yielding recAb with sequences similar to the $\mathrm{BCR}$ of the naive $\mathrm{B}$-cell receptor ( $\mathrm{B} B \mathrm{CR}$ ) from which the tumor cells originated, to obtain further insight into the impact of SHM on BCR characteristics of PCNSL. To this end, we focused on IGHV4-34+ and IGHV3 ${ }^{+}$PCNSL, as the IGHV4-34 gene is preferentially rearranged in PCNSL, while genes of the large IGHV3 subgroup also occur frequently, but without biased usage. ${ }^{2-4}$ Comparison of $\mathrm{nBCR}$ with $\mathrm{tBCR}$ reactivity demonstrated that autoreactivity and polyreactivity increased upon SHM. Thus, in PCNSL, B cells exhibit features that, principally, qualify for elimination; however, they are rescued by paradoxical redemption in the GC reaction.

\section{Methods}

\section{Patients}

Stereotactic PCNSL biopsies (tumor load $>80 \%$ ) from 50 HIVnegative patients were included (Online Supplementary Table S1). PCNSL were diagnosed according to World Health Organization guidelines. ${ }^{1}$ The study was approved by the Ethics Committee of the University Hospital of Cologne (06-187, 07-109) and performed according to the Declaration of Helsinki.

\section{Tumor B-cell receptor reversion}

TBCR sequences (PCNSL patients \#01 - \#10) (Online Supplementary Tables $S 1$ and $S 2)^{10}$ were reverted to their unmutated form using the ImMunoGeneTics (IMGT) information system database ( $w$ ww. imgt.org ${ }^{11}$ ) similar to the approach reported by Herve et al. ${ }^{12}$ reverting V-derived sequences of the VH and VK/VL domain. Mutated nucleotides were replaced by those in the variant region being most similar to the germline gene segment according to IMGT, which was considered as naïve sequence. These sequences were synthetized by GeneArt (Thermo Fisher Scientific, Rockford, IL, USA); correctness was controlled by Sanger sequencing.

\section{Generation of recombinant antibodies with binding specificity identical to tumor B-cell and naïve B-cell receptors}

Monoclonal recAb with binding specificity identical to the $\mathrm{tBCR}$ and their corresponding $\mathrm{nBCR}$ were generated as described ${ }^{10}$ (Online Supplementary Figure S1). Ig V gene sequences are detailed in Online Supplementary Table S3.

As control, the recombinant anti-tetanus toxoid antibody recSA13 was generated from the SA13 cell line (HB8501, LCG Standard, Wesel, Germany) using the same strategy. ${ }^{10}$ For specificity of recSA13 see Online Supplementary Table S4.

\section{Protein microarray processing}

Protein microarray slides (9374 human proteins, ProtoArray, Thermo Fisher Scientific) including internal controls were analyzed as described previously. ${ }^{10,13,14}$

\section{Immunohistochemistry}

RecAb of PCNSL patients \#01, \#03, and \#09 labeled with green fluorescent dye CF488A (Antibody Labeling Kit, Sigma, Deisenhofen, Germany), were applied to PCNSL sections, followed by incubation with monoclonal rabbit anti-SNRPC (clone EPR16034, Abcam, Cambridge, UK) and species-specific Cy3-coupled immunoglobulin (Dianova, Hamburg, Germany).

Double immunofluorescence was performed with rabbit antiSNRPC and mouse anti-GFAP (clone GA-5, Biogenex, Fremont, CA, USA), mouse anti-Olig2 (clone OLIG2/2400, Abcam), mouse anti-NeuN (clone A60, Merck, Burlington, MA, USA), mouse antiCD68 (clone KP1, DCS, Hamburg, Germany), and mouse antiCD34 (clone Obend/10, Biogenex, Palo Alto, CA, USA), respectively, including appropriate positive and negative controls (Online Supplementary Figures S2 and S3) was performed.

\section{Analysis of gene expression profiling}

Gene expression profiling (GEP) (10 normal CNS tissues, 21 PCNSL) obtained by the Affymetrix U95Av2 microarray (Santa Clara, CA, USA) published previously ${ }^{15}$ were analyzed for SNRPC mRNA expression.

\section{Statistical analysis}

To test for statistical significance between the number of proteins recognized by the recAb derived from $\mathrm{nBCR}$ and $\mathrm{tBCR}$, the exact Wilcoxon signed rank test (calculated with $\mathrm{R}$ version 3.5.0, $\mathrm{R}$ Foundation for Statistical Computing, Vienna, Austria) was applied. Statistical significance between SNRPC mRNA expression in normal CNS and PCNSL was assessed by Student $t$-test. $P<0.05$ was considered significant. All experiments were performed at least in duplicate.

\section{Results}

Immunoglobulin heavy and light chain variable gene rearrangements in primary lymphoma of the central nervous system used for generation of recombinant antibodies

All PCNSL harbored a monoclonally rearranged Ig heavy chain variable and a monoclonally rearranged Ig light chain variable gene. A gene of the IGHV3 and the IGHV4 subgroup was rearranged in five tumors each with 
Table 1. Autoreactivity of the recombinant antibodies tested by HEp-2 immunofluorescence, ANAcombi and ANCAcombi ELISA.

\begin{tabular}{|c|c|c|c|c|c|c|c|}
\hline \multirow{2}{*}{$\begin{array}{l}\text { PCNSL } \\
\text { Patient n. }\end{array}$} & \multirow[t]{2}{*}{ IEHV } & \multicolumn{2}{|c|}{ ANA-HEp-2 } & \multicolumn{2}{|c|}{ ANAcombi } & \multicolumn{2}{|c|}{ ANCAcombi } \\
\hline & & $\mathrm{nBCR}$ & tBCR & nBCR & tBCR & nBCR & tBCR \\
\hline$\# 01$ & IGHV3-23 & - & + & - & - & - & - \\
\hline$\# 02$ & IGHV3-30 & + & + & - & - & - & - \\
\hline$\# 03$ & IGHV3-48 & - & - & - & - & - & - \\
\hline$\# 04$ & IGHV3-7 & - & - & - & - & - & - \\
\hline$\# 05$ & IGHV3-74 & - & + & - & - & - & - \\
\hline$\# 06$ & IGHV4-34 & - & - & - & - & - & - \\
\hline$\# 07$ & IGHV4-34 & - & + & - & - & - & - \\
\hline$\# 08$ & IGHV4-34 & - & + & - & $+a$ & - & $+b$ \\
\hline$\# 09$ & IGHV4-34 & - & - & - & - & - & - \\
\hline$\# 10$ & IGHV4-34 & - & - & - & - & $+c$ & $+d$ \\
\hline
\end{tabular}

a: RNP-70 (small nuclear ribonucleoprotein U1 subunit 70); b: lysozyme; c: lysozyme, elastase, myeloperoxidase, BPI (bactericidal permeability-increasing protein); d: lysozyme, elastase, proteinase 3. PCNSL: primary central nervous system lymphoma; nBCR: naïve B-cell receptor; tBCR: tumor cell B-cell receptor.

five distinct members of the IGHV3 subgroup and five IGHV4-34+ PCNSL (Online Supplementary Table S2)..$^{10}$ All PCNSL had introduced somatic mutations into their rearranged Ig heavy and light chain variable genes. All Ig heavy and light chain sequences harbored mutations varying from $2-15 \%$ with mean mutation frequencies of $9.9 \%$ and $8.3 \%$ for the heavy and the light chain V-genes, respectively. Mutations corresponded to point mutations in the heavy and light chain of all ten PCNSL. In addition, case \#01 harbored an insertion of six base pairs which extended the heavy chain CDR2 from eight to ten amino acids (Online Supplementary Table S3). As we attributed this insertion to SHM, it was eliminated in the revertant sequence (Online Supplementary Table S3). Deletions and stop codons were absent. Thus, all tumors harbored rearranged and somatically mutated Ig heavy and light chain variable genes encoding a potentially functional $\mathrm{BCR}$, in accordance with previous data. ${ }^{2-4}$

\section{Recombinant antibodies of naïve and tumor B-cell receptor show self-reactivity with common autoantigens}

Complete IgG recAb of $\mathrm{nBCR}$ and $\mathrm{tBCR}$ were compared for reactivity with common self-antigens using permeabilized human HEp-2 cells as target. In five cases, a positive reaction indicated autoreactivity (Table 1 and Online Supplementary Figure S4). In four of them, the SHMinduced modification of the nBCR yielded tBCR HEp-2 reactivity not noticed for the $\mathrm{nBCR}$ (Table 1 ). In the remaining case, both $\mathrm{NBCR}$ and $\mathrm{tBCR}$ were $\mathrm{HEp}-2$ reactive (Table 1). None of the $\mathrm{nBCR}$ exhibited reactivity that was lost upon SHM. Remarkably, HEp-2 reactivity was much below the standard (Online Supplementary Figure S4), detectable at high, but not low recAb concentration $(250 \mu \mathrm{g} / \mathrm{mL}$ vs. $10 \mu \mathrm{g} / \mathrm{mL})$, indicating low affinity of the rec $A B$.

ANA/ANCA ELISA detected reactivity in two IGHV4$34^{+}$PCNSL (Table 1). In one case (PCNSL patient \#10), both $\mathrm{nBCR}$ and $\mathrm{tBCR}$ reacted with autoantigens, while in the other case (PCNSL patient \#08) only the tBCR, but not the $\mathrm{nBCR}$ was reactive (Table 1). Again, BCR binding was much below the standard of the ELISA requiring a high antibody concentration $(200 \mu \mathrm{g} / \mathrm{mL})$. At a concentration recommended for the detection of high-affinity antibodies in patients with clinically active autoimmune disease $(10 \mu \mathrm{g} / \mathrm{mL})$, in this regard differing from PCNSL-derived
$\mathrm{nBCR}$ and $\mathrm{tBCR}$, reactivity was completely lost, further suggesting low affinity. Together, these data show that the rec $A B$ of 6 of $10(60 \%)$ PCNSL exhibit weak reactivity with self-antigens, mostly in the $\mathrm{tBCR}$, indicating that SHM fosters self-reactivity.

\section{Naïve and tumor B-cell receptor differ in their protein recognition pattern on the ProtoArray}

All recAb specifically recognized proteins on the ProtoArray. A total of 821 proteins was specifically recognized by at least one recAb (mean: 165; median: 140; range: 106-300). Regarding all proteins recognized, 341 and 725 proteins were recognized by at least one of the $\mathrm{nBCR}$ and $\mathrm{tBCR}$, respectively. The $\mathrm{nBCR}$ exclusively recognized 96 proteins including several ribosomal proteins, B-cell linker, and ligase III (Online Supplementary Table S5), whereas the $\mathrm{tBCR}$ exclusively recognized 480 proteins including histones, myelin associated proteins (i.e., myelin basic protein, MBP; myelin-associated oligodendrocyte basic protein, MOBP; myelin protein zero-like protein 1 , MPZL1), ribosomal proteins, and splicing factors (SNRP70, SNRPA, SNRPB) (Online Supplementary Table S5). A total of 245 proteins were recognized by both the $\mathrm{nBCR}$ and the tBCR including galectins, S100A1 protein, and SNRPC (Online Supplementary Table S4). With respect to the proteins recognized by the $\mathrm{nBCR}, 107$ and 58 proteins were recognized exclusively by the $\mathrm{nBCR}$ derived from $\mathrm{IGHV3}^{+}$and IGHV4-34+ PCNSL, respectively; 176 proteins were recognized in common. Regarding the proteins recognized by the tBCR, 248 and 179 proteins were recognized exclusively by the tBCR derived from $\mathrm{IGHV}^{+}$and IGHV4-34+ PCNSL, respectively; 298 proteins were recognized in common (Figure 1A and Online Supplementary Table S5).

According to the Human Protein Atlas Project (www.proteinatlas.org), 482 proteins recognized by the recAb are expressed in the CNS, albeit mostly not exclusively (mean: 96; median: 82; range: 63-184). Of those CNS proteins recognized, 202 and 425 CNS proteins were recognized by at least one of the $\mathrm{NBCR}$ and the $\mathrm{ABCR}$, respectively (Figure $1 B$ ). NBCR exclusively recognized 57 proteins including BLNK and LIG23 (Online Supplementary Table S5), whereas tBCR exclusively recognized 280 proteins including MBP, MOBP, MPZL1, SNRPA, SNRPB2, and S100A13 (Online Supplementary Table S5); 145 proteins were recognized in common including endoglin, galectins, 
A

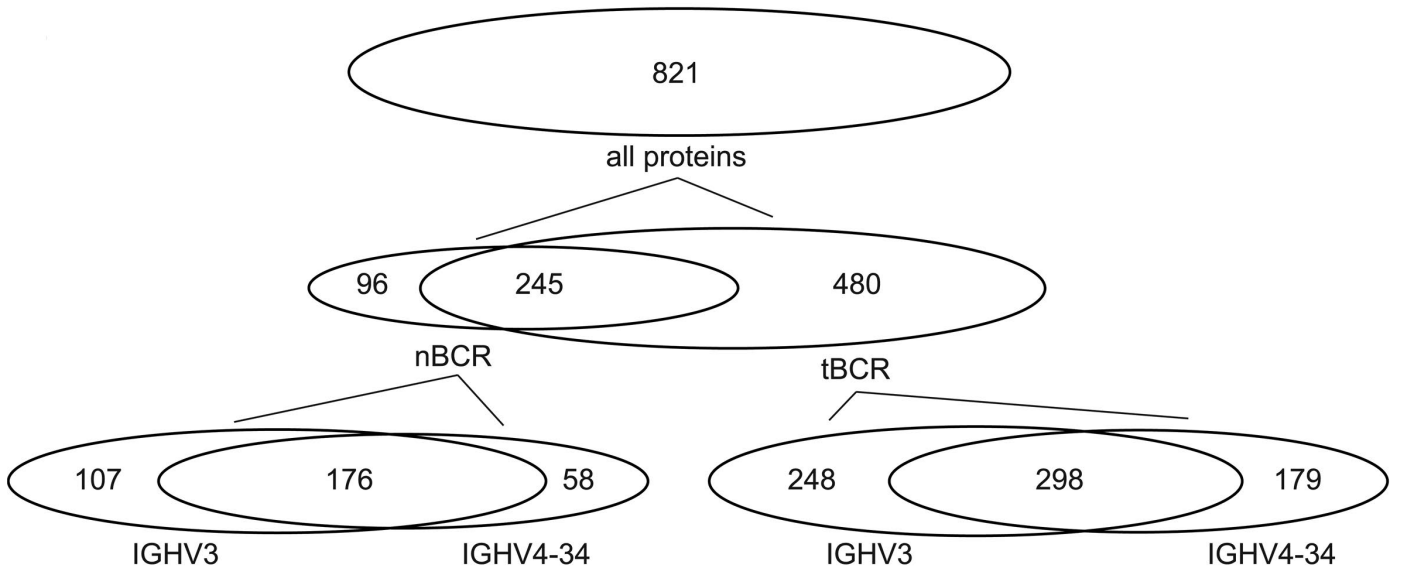

B

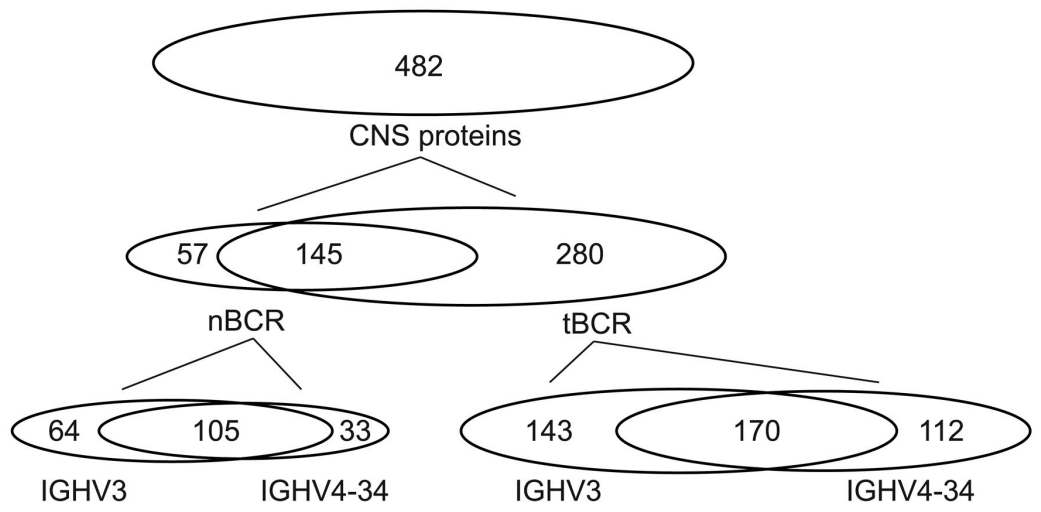

Figure 1. Recognition pattern of recombinant antibodies (recAb) derived from naïve B-cell receptor ( $\mathrm{BBCR}$ ) and tumor $\mathrm{B}$-cell receptor (tBCR) of primary lymphoma of the central nervous system (PCNSL) on the ProtoArray. (A) Quantitative Venn diagrams show numbers of proteins recognized by at least one recAb. The numbers of proteins recognized exclusively by $\mathrm{nBCR}$ or $\mathrm{HBCR}$ are shown in the middle panel. The bottom panel depicts numbers of proteins recognized exclusively by $\mathrm{nBCR}$ or tBCR derived from IGHV3 ${ }^{+}$or IGHV4-34 $4^{+}$PCNSL. (B) Quantitative Venn diagrams show numbers of CNS proteins recognized by at least one recAb (upper panel). The numbers of CNS proteins recognized exclusively by $\mathrm{BBCR}$ or $\mathrm{HBCR}$ are shown in the middle panel. The bottom panel depicts numbers of CNS proteins recognized exclusively by $\mathrm{nBCR}$ or tBCR derived from either IGHV3+ or IGHV4-34+ PCNSL.

and SNRPC (Online Supplementary Table S5). Regarding CNS proteins recognized by $\mathrm{nBCR}, 64$ and 33 CNS proteins were exclusively recognized by $\mathrm{nBCR}$ derived from $\mathrm{IGHV3}^{+}$and IGHV4-34+ PCNSL, respectively; 105 proteins were recognized in common (Figure $1 \mathrm{~B})$. Regarding CNS proteins recognized by $\mathrm{tBCR}, 143$ and $112 \mathrm{CNS}$ proteins were exclusively recognized by the $\mathrm{tBCR}$ derived from $\mathrm{IGHV3}^{+}$and IGHV4-34 $4^{+} \mathrm{PNSL}$, respectively; 170 proteins were recognized in common (Online Supplementary Table S5).

Focusing on individual PCNSL, tBCR of both $\mathrm{IGHV}^{+}$ and IGHV4-34+ PCNSL recognized significantly increased numbers of proteins compared to nBCR (Figure 2A-D). Proteins recognized exclusively by $\mathrm{tBCR}$ expressed in the CNS included histones, ribosomal proteins, CNS hormones (arginine vasopressin-induced 1, neuropeptide Y), proteins involved in glial cell metabolism (S100-A13, MPZL1, MBP, MOBP), and mitochondria (ATP/ATPases, cytochrome C) (Online Supplementary Table S5).

Thus, nBCR alter their protein recognition pattern with B-cell maturation. In addition to proteins recognized by both $\mathrm{nBCR}$ and $\mathrm{tBCR}$ and those exclusively bound by $\mathrm{tBCR}$, we also detected proteins that were recognized by the $\mathrm{nBCR}$ and lost reactivity with modification towards the $\mathrm{tBCR}$. Importantly and of potentially functional relevance, the BCR increases its protein reactivity upon SHM enabling recognition of increased numbers of antigens expressed in the CNS.

\section{Reactivity of naïve and tumor B-cell receptor with central nervous system proteins determined by immunoprecipitation}

Normal brain biopsies were used for immunoprecipitation with all recAb. A total of 270 proteins (mean: 80.2, median: 82.5 , range: 44-124) co-immunoprecipitated with at least one recAb (Figure 3A); 214 and 241 proteins coimmunoprecipitated with at least one $\mathrm{nBCR}$ and $\mathrm{tBCR}$, respectively. NBCR and $\mathrm{ABCR}$ exclusively immunoprecipitated 29 and 56 proteins, respectively; 185 proteins were recognized in common. Regarding $\mathrm{nBCR}$ corresponding to IGHV3 $^{+}$and IGHV4-34+ PCNSL, 59 and 42 proteins were exclusively immunoprecipitated, respectively; 113 were recognized in common. With respect to the $\mathrm{tBCR}, 47$ proteins each were immunoprecipitated exclusively by IGHV3 $^{+}$and IGHV4-34 ${ }^{+}$PCNSL; 147 proteins were immunoprecipitated in common (Figure $3 \mathrm{~A}-\mathrm{C}$ ). 
A

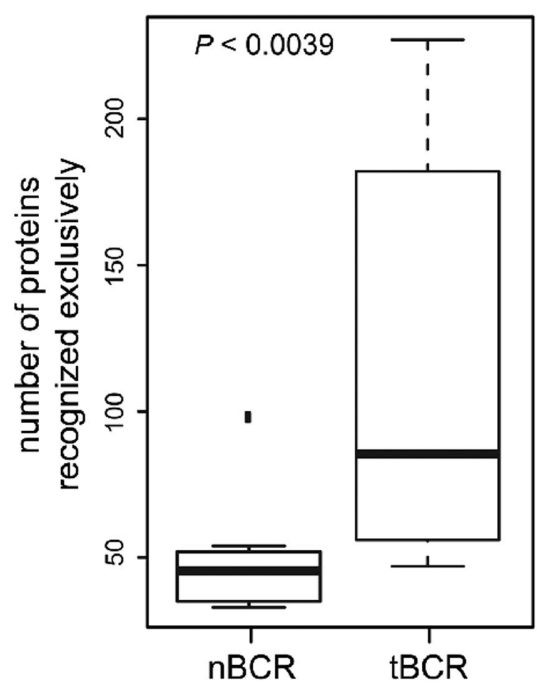

C

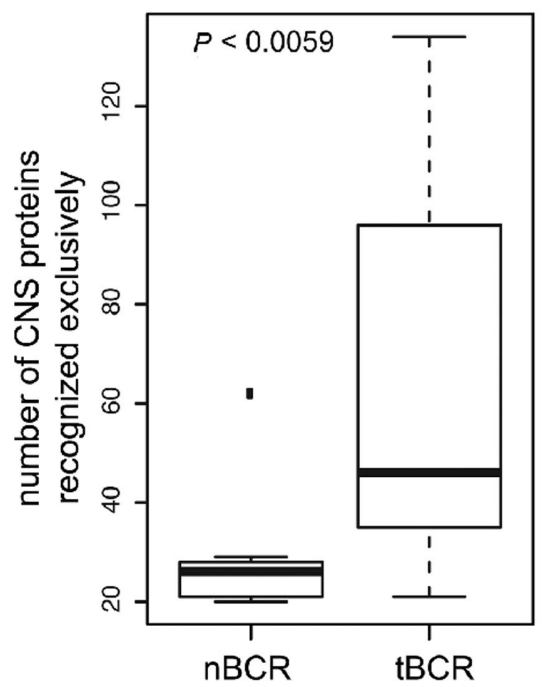

B

\begin{tabular}{lcccc}
\hline IGHV & case & nBCR & nBCR+tBCR & tBCR \\
\hline IGHV3-23 & $\# 01$ & 33 & 73 & 227 \\
IGHV3-30 & $\# 02$ & 98 & 84 & 182 \\
IGHV3-48 & $\# 03$ & 35 & 82 & 80 \\
IGHV3-7 & $\# 04$ & 48 & 86 & 47 \\
IGHV3-74 & $\# 05$ & 39 & 86 & 94 \\
\hline IGHV4-34 & $\# 06$ & 50 & 81 & 56 \\
IGHV4-34 & $\# 07$ & 54 & 103 & 91 \\
IGHV4-34 & $\# 08$ & 52 & 91 & 209 \\
IGHV4-34 & $\# 09$ & 43 & 84 & 73 \\
IGHV4-34 & $\# 10$ & 34 & 85 & 47 \\
\hline
\end{tabular}

D

\begin{tabular}{lcccc}
\hline IGHV & case & nBCR & nBCR+tBCR & tBCR \\
\hline IGHV3-23 & $\# 01$ & 20 & 43 & 134 \\
IGHV3-30 & $\# 02$ & 62 & 47 & 96 \\
IGHV3-48 & $\# 03$ & 21 & 50 & 41 \\
IGHV3-7 & $\# 04$ & 28 & 51 & 21 \\
IGHV3-74 & $\# 05$ & 27 & 50 & 55 \\
\hline IGHV4-34 & $\# 06$ & 24 & 49 & 35 \\
IGHV4-34 & $\# 07$ & 29 & 60 & 51 \\
IGHV4-34 & $\# 08$ & 28 & 52 & 132 \\
IGHV4-34 & $\# 09$ & 25 & 53 & 40 \\
IGHV4-34 & $\# 10$ & 21 & 48 & 25 \\
\hline
\end{tabular}

Figure 2. Recognition pattern of recombinant antibodies (recAb) derived from naïve $B$ cell receptor ( $\mathrm{nBCR}$ ) and tumor cell $\mathrm{B}-\mathrm{cell}$ receptor (tBCR) receptor of primary lymphoma of the central nervous system (PCNSL). (A) BoxPlot diagram of ProtoArray analysis showing numbers of proteins recognized by at least one nBCR-derived recAb or tBCR-derived recAb. Numbers of proteins recognized exclusively by either nBCR or tBCR in individual PCNSL were used for calculation. Statistical significance was determined by exact Wilcoxon signed rank test. (B) Number of proteins on the ProtoArray that are recognized by recombinant antibodies derived from nBCR and tBCR are shown for individual PCNSL cases. In addition to proteins recognized by either nBCR- or tBCR-derived recAbs, the number of shared target proteins is depicted. (C) BoxPlot diagram shows the numbers of proteins known to be expressed in the central nervous system (CNS) that were recognized by at least one nBCR and tBCR recAb. Proteins known to be expressed in the CNS recognized exclusively by either $n B C R$ or tBCR in individual PCNSL were used for calculation. Statistical significance was determined by exact Wilcoxon signed rank test. (D) The number of proteins on the ProtoArray known to be expressed in the CNS that are recognized by recombinant antibodies derived from $\mathrm{nBCR}$ and $\mathrm{tBCR}$ is shown for individual PCNSL cases. In addition to proteins recognized by either nBCR- or tBCR-derived recombinant antibodies, the number of shared target proteins is depicted.

While a lack of information on protein characteristics on the ProtoArray and within human brain samples regarding isoform(s) and post-translational modifications hampers direct comparison of the proteins identified in these two assays, both techniques led to the conclusion that tBCR recognize significantly increased numbers of CNS proteins and identified proteins recognized exclusively by the tBCR. Among the proteins differentially recognized by the $\mathrm{tBCR}$, but not the $\mathrm{nBCR}$, were ribosomal proteins, histones, and proteins involved in mitochondrial and glial cell metabolism (laminin subunit $\beta-1$, annexin A7, protein S100-A9) (Online Supplementary Table S6).

Immunoprecipitation of the $\mathrm{nBCR}$ and $\mathrm{tBCR}$ with
PCNSL lysates was limited due to tissue scarcity with low protein content; nevertheless, there was a tendency towards increased numbers of proteins recognized by tBCR compared to $\mathrm{nBCR}$, which, however, did not reach statistical significance (Online Supplementary Figure S5). $\mathrm{RecAb}$ binding in dot blots confirmed that western blot conditions were too stringent (Online Supplementary Figures S6 and S7).

Immunofluorescence studies confirm binding of recombinant antibodies derived from naïve and tumor B-cell receptor of proteins recognized on the ProtoArray

To confirm the ProtoArray data by an independent 


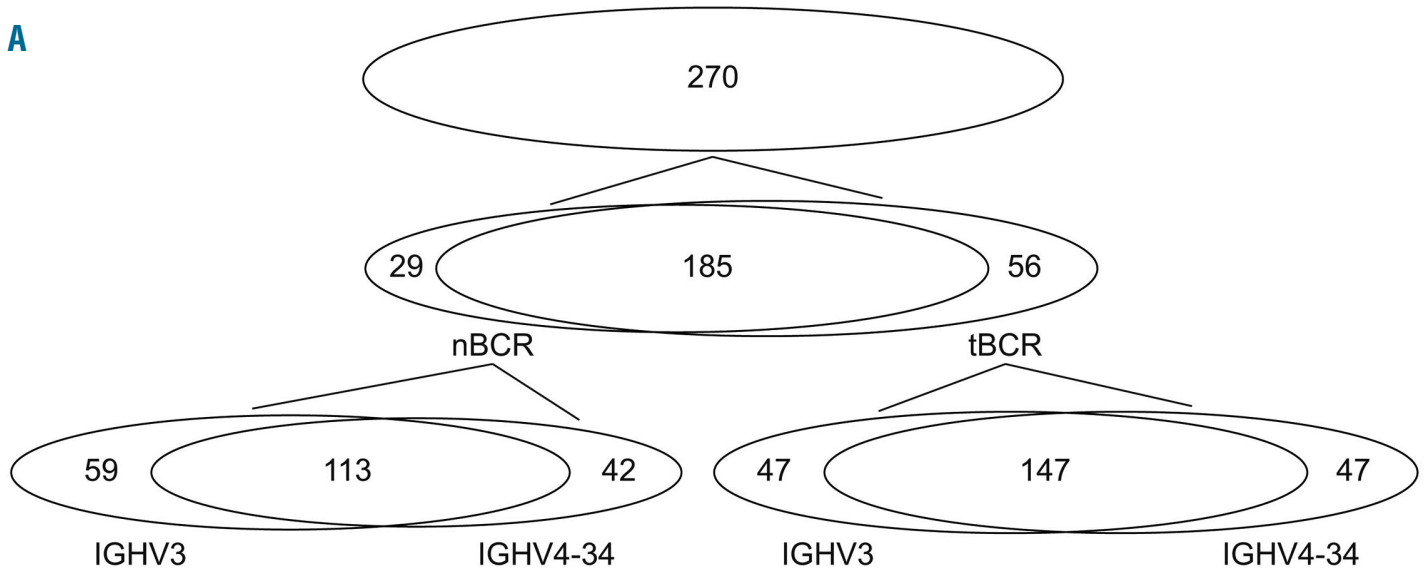

B

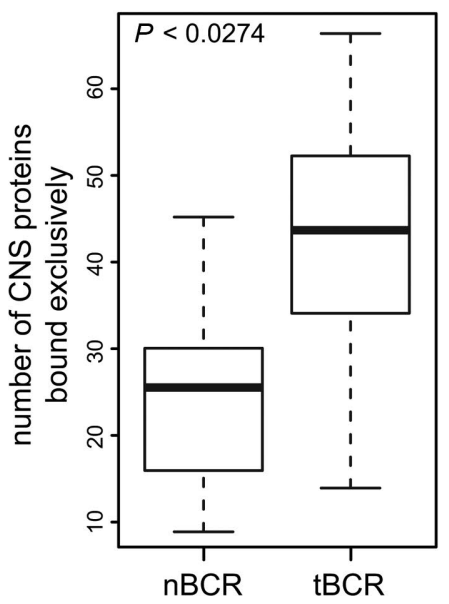

C

\begin{tabular}{lcccc}
\hline IGHV & case & nBCR & nBCR+tBCR & tBCR \\
\hline IGHV3-23 & $\# 01$ & 29 & 48 & 29 \\
IGHV3-30 & $\# 02$ & 20 & 42 & 52 \\
IGHV3-48 & $\# 03$ & 45 & 41 & 38 \\
IGHV3-7 & $\# 04$ & 29 & 52 & 46 \\
IGHV3-74 & $\# 05$ & 30 & 56 & 57 \\
\hline IGHV4-34 & $\# 06$ & 16 & 30 & 14 \\
IGHV4-34 & $\# 07$ & 13 & 58 & 66 \\
IGHV4-34 & $\# 08$ & 9 & 49 & 41 \\
IGHV4-34 & $\# 09$ & 31 & 53 & 34 \\
IGHV4-34 & $\# 10$ & 22 & 39 & 48 \\
\hline
\end{tabular}

Figure 3. Tumor cell B-cell receptors (tBCR) recognize increased numbers of proteins in the central nervous system (CNS) as compared to naïve B-cell receptors (nBCR) in immunoprecipitation studies. (A) Quantitative Venn diagrams of immunoprecipitation analysis show the number of proteins that co-immunoprecipitate with at least one recombinant antibody (recAb) (upper panel). The middle panel shows numbers of proteins co-immunoprecipitating exclusively with nBCR or tBCR. The bottom panel depicts numbers of proteins co-immunoprecipitating nBCR or tBCR derived from either IGHV3 ${ }^{+}$or IGHV4-34 ${ }^{+}$primary lymphoma of the central nervous system (PCNSL). Proteins co-immunoprecipitating with both recAb are shown in the intersection. (B) BoxPlot diagram of immunoprecipitation shows the numbers of proteins co-immunoprecipitating with at least one recAb. Only proteins co-immunoprecipitating with either nBCR or tBCR were analyzed. Statistical significance was determined by exact Wilcoxon signed rank test. (C) Numbers of proteins co-immunoprecipitating with nBCR and tBCR shown for individual PCNSL cases. In addition, numbers of shared target proteins are depicted.

method, double immunofluorescence of SNRPC expression was performed.

SNRPC was differentially recognized by recAb derived from $\mathrm{nBCR}$ and $\mathrm{tBCR}$ on the ProtoArray (nBCR: negative, tBCR: positive, PCNSL patients \#01, \#03, \#09). RecAB derived from the $\mathrm{nBCR}$ of PCNSL patients \#01, \#03, and \#09 did not co-localize with the commercial anti-SNRPC antibody (Figure 4A-C). In contrast, the rec $\mathrm{Ab}$ derived from the $\mathrm{tBCR}$ co-localized with the commercial anti-SNRPC antibody demonstrating tumor cell SNRPC expression (Figure 4D-F). RecAb staining was remarkably less strong compared to the commercial monoclonal anti-SNRPC antibody, further indicating their lower affinity. This observation was further supported by ELISA analysis of the recAb compared to the commercial anti-SNRPC antibody (Online Supplementary Figure S8). Here, only a very high concentration of the $\mathrm{rec} \mathrm{Ab}$ together with a much lower concentration of the commercial anti-SNRPC antibody revealed reactivity (Online Supplementary Figure S8).

Thus, these in situ topographical proof-of-principle analyses confirmed the ProtoArray data further supporting low-affinity and polyreactivity of $\mathrm{nBCR}$ and $\mathrm{tBCR}$.

\section{Proteins recognized by recombinant antibodies are expressed in primary lymphoma of the central nervous system}

Topographical analysis revealed that 90\% (18 of 20) of PCNSL (PCNSL patients \#11 - \#30). showed a nuclear SNRPC expression in the majority of the tumor cells (Figure 5A and Online Supplementary Table S7). Neurons, astrocytes, oligodendrocytes, a few microglial cells and macrophages also expressed SNRPC (Figure 5B-F). GEP ${ }^{15}$ confirmed a significantly increased SNRPC expression in PCNSL compared to normal brain (Figure 5G).

\section{Discussion}

This study demonstrates that reverting somatic mutations of the V-derived sequences of the $\mathrm{VH}$ and $\mathrm{VK} / \mathrm{L}$ domain of PCNSL tBCR can yield a low-affinity, self- and 

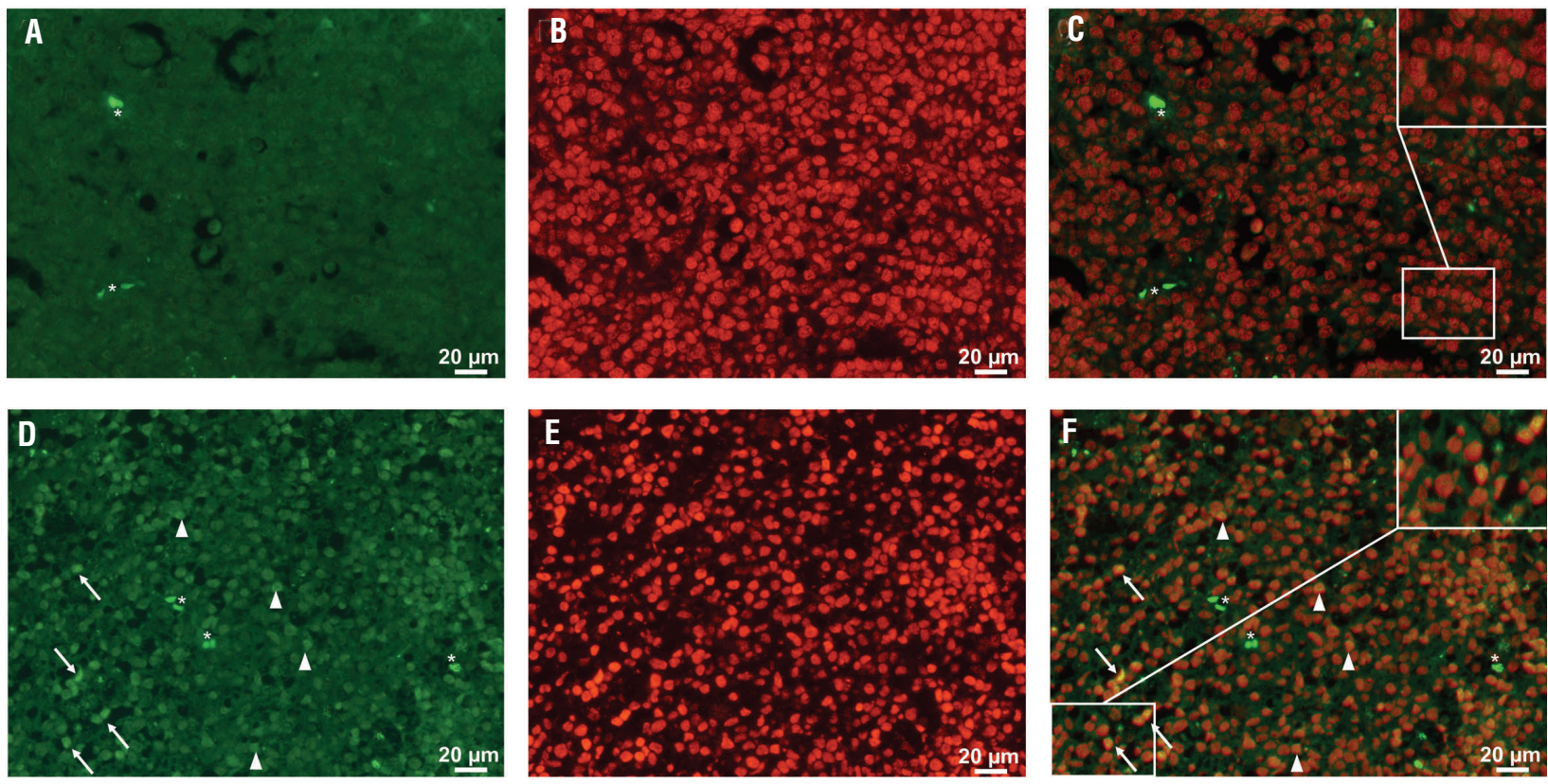

Figure 4. Recognition of SNRP by recombinant antibodies (recAb) corresponding to naïve B-cell receptors (nBCR) and tumor cell receptors (tBCR). (A-C) In primary lymphoma of the central nervous system (PCNSL) patient \#17, the nBCR (FITC, A) derived from PCNSL patient \#09, which did not react with SNRPC on the ProtoArray (Online Supplementary Table S4), shows only a weak staining of single tumor cells (arrows, A). Prominent expression of SNRPC in the nuclei of the majority of tumor cells in PCNSL patient \#17 is evidenced by application of the commercial anti-SNRPC antibody (Cy3, B). The overlay (C) shows that there is no co-localization of both antibodies (arrows, C). Insert: high-power magnification of (C) x1000. *Erythrocyte autofluorescence. (D-F) tBCR (FITC, D) derived from PCNSL patient \#09, which reacted with SNRPC on the ProtoArray (Online Supplementary Table S4), co-localizes with the commercial anti-SNRPC antibody (Cy3, E and F) that depicts prominent SNRPC expression by the tumor cells of PCNSL patient \#17 (E). Arrows and arrowheads in (D) indicate prominent and moderate immunoreactivity of the tBCR, respectively, yielding a yellow (arrows, F) or orange (arrowheads, F) signal in the tumor cell nuclei in the overlay (F). Insert: high-power magnification of F, x1000. *Erythrocyte autofluorescence. Double immunofluorescence using rabbit anti-SNRPC (clone EPR16034, Abcam, Cy3) with nBCR (A) and tBCR (D) labeled with Mix'n Stain 488 Labeling Kit (Sigma). Microphotographs were taken with an Axiophot (Zeiss) and Zen 2 software (Zeiss). Original magnification $x 400$ (objective: $x 40)$. Overlay of the microphotographs ( $\mathrm{C}$ and $\mathrm{F}$ ) and adjustment for contrast and brightness were performed with Adobe Photoshop software version CC. Similar results were obtained for staining with recAb derived from PCNSL patients \#03 and \#09, as well as for staining of sections derived from PCNSL patients \#14, \#21, and \#24.

polyreactive BCR of the naïve precursor B cells. Comparison of the protein recognition pattern of $\mathrm{nBCR}$ and $\mathrm{tBCR}$ reveals increased polyreactivity of the GC-experienced $B$ cell with a particularly increased reactivity with proteins expressed in the CNS.

Gain of self- and polyreactivity during SHM was supported by several experiments, i.e., reactivity with selfantigens in immunofluorescence with HEp-2 cells, ANA/ANCA ELISA at high, but not low BCR concentration, ProtoArray analysis, immunoprecipitation, and topographical immunohistochemistry. Comparability between the various methods is hampered by technical limitations due to specific characteristics of the respective analyses, e.g., different isoforms of proteins expressed may be present in the human brain and on the ProtoArray, a lack of knowledge of post-translational protein modifications and other metabolic modifications in the human brain. It is of note that our recAbs were expressed as IgG, a well-established technique frequently used by many groups. ${ }^{16,17}$ Recent studies demonstrated that the in vitro binding of at least certain polyreactive Igs differed from the IgM-BCR counterpart, ${ }^{18}$ that the $\mathrm{C}$ region may change the fine antibody specificity for particular antigens, ${ }^{19}$ and that the isotype shapes epitope specificity, antibody affinity, and functional activities. ${ }^{20}$ Thus, one cannot exclude with certainty that some specificities may be confined to the soluble IgG antibody. However, it is unlikely that this holds true for all of the many specificities of all recAbs of our series. Moreover, the fact that the IgG generated from the tBCR did not show the same autoreactivity as their corre- sponding IgG generated from the $\mathrm{nBCR}$, further supports the concept that the expression of an IgM BCR as a soluble IgG antibody does not systematically lead to a gain of autoreactivity and hence serves as a kind of internal control. $\mathrm{N}$ - and P-nucleotides of the CDR3 sequence cannot be reverted due to lack of corresponding germline sequences. However, it is unlikely that mutations persisting in CDR3, which can also modify antibody affinity and specificity, may affect the overall results of the entire PCNSL series, in particular, as PCNSL are characterized by unrestricted CDR3 length and amino acid composition and by an absence of stereotyped BCR sequences. ${ }^{4}$ Nevertheless, despite these technical limitations, all analyses indicated increased poly-/autoreactivity of the tBCR and a faulty GC reaction, thus extending previous data on polyreactive BCR of PCNSL and an involvement of numerous antigens in tumor cell selection. ${ }^{4,10,21}$

These observations fit into the concept that antigen selection plays an important role in driving BCR activity in PCNSL. There is growing evidence to suggest that BCR signaling in response to self and/or foreign antigens present in the target organ microenvironment sustains BCR signaling in various mature B-cell lymphoma entities including PCNSL, follicular lymphoma, splenic marginal zone lymphoma, mucosa-associated lymphoid tissue lymphoma of the eye, and chronic lymphocytic leukemia (CLL). ${ }^{10,12,16,22,23}$ In CLL, B cells with unmutated BCR express highly polyreactive antibodies whereas most mutated CLL B cells do not. ${ }^{12}$ Mutated non-autoreactive CLL antibody sequences reverted into their germline 

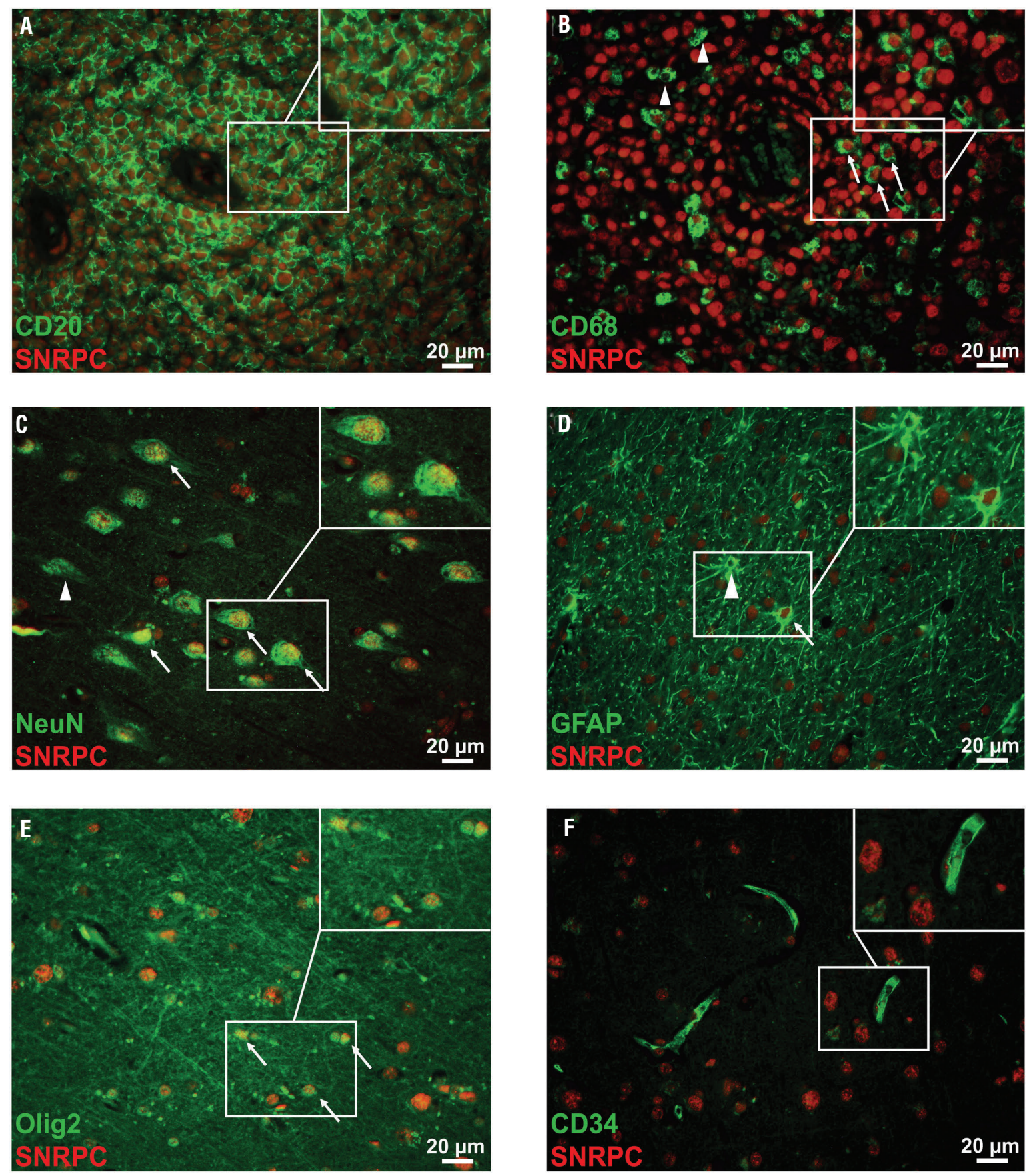

G

CNS

PCNSL

\section{SNRPC}
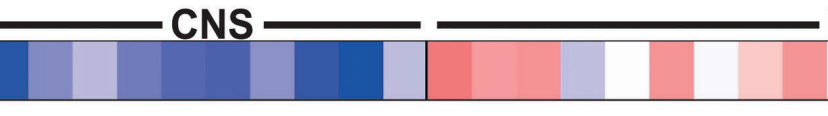

Figure 5. Expression of SNRPC in primary lymphoma of the central nervous system (PCNSL). (A) Nearly all CD20 (FITC) tumor cells of PCNSL show a nuclear expression of SNRPC (Cy3). (B) Intermingled with tumor cells of a PCNSL, single CD68 ${ }^{+}$(FITC) macrophages also express SNRPC (arrows, Cy3); additionally, there are also

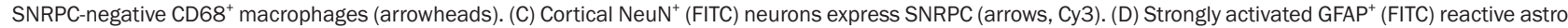
cytes express SNRPC (arrows, Cy3). (E) In the white matter, some Olig2 ${ }^{+}$(FITC) oligodendrocytes express SNRPC (arrows, Cy3). (F) CD34 ${ }^{+}$(FITC) endothelial cells of neocortical cerebral blood vessels do not express SNRPC (Cy3). (G) mRNA expression of SNRPC recognized on the ProtoArray in the normal central nervous system (CNS) and PCNSL. SNRPC is downregulated CNS and upregulated in PCNSL samples. The line represents the SNRPC tag on the U95Av2 microarray (Affymetrix) in individual samples of ten normal CNS tissues and 21 PCNSL published previously. ${ }^{15}$ SNRPC was significantly differentially expressed between CNS and PCNSL $(P<0.01$, Student $t$-test). (A-F) Double immunofluorescence with rabbit anti-SNRPC (clone EPR16034, Abcam, Cy3) and mouse anti-CD20 (clone L26, DCS, FITC in A), mouse anti-CD68 (clone KP1, DCS, FITC in B), mouse anti-NeuN (clone A60, Millipore, FITC in C), mouse anti-GFAP (clone GA-5, Biogenex, FITC in D), mouse antiOlig2 (clone Olig2/2400, Abcam, FITC in E), and mouse anti-CD34 (cloneQBend/10, Biogenex, FITC in F). Microphotographs were taken with an Axiophot (Zeiss, Jena, Germany) and Zen 2 software (Zeiss). Original magnification: x400 (objective: x40). Inserts: x750 (A-C), x1000 (D-F). Overlay of the microphotographs and adjustment for contrast and brightness were performed with Adobe Photoshop software version CC. 
counterparts' encoded poly-/autoreactive antibodies, indicating that SHM plays an important role in disease development by altering original BCR autoreactivity and negatively selecting for auto-/polyreactivity. ${ }^{12}$ In this regard, SHM performs its physiological function in B-cell differentiation to eliminate self-reactive $\mathrm{B}$ cells. ${ }^{17} \mathrm{SHM}$ also strongly impacts on BCR reactivity in PCNSL, however, with opposite results, at least in some instances. In PCNSL, a failure of the selection process in the GC reaction may be a crucial event broadening autoantigenic reactivity, thereby fostering survival upon antigen encounter.

Physiologically, low-affinity, autoreactive B cells with $\mathrm{IgD}^{+} \mathrm{IgM}^{+}$immunophenotype, a hallmark of PCNSL, ${ }^{24}$ are anergic, short-lived and have long been considered to be excluded from the GC. ${ }^{25}$ However, it was recently shown that in particular conditions autoreactive B cells may enter a GC reaction, but lose their autoreactivity as a result of SHM. ${ }^{26}$ Such a mechanism has also been reported for B cells using the IGHV4-34 gene that often encodes autoreactive antibodies. ${ }^{2}$ PCNSL also preferentially rearrange the IGHV4-34 gene..$^{2-4}$ On the other hand, B cells can acquire poly-/self-reactivity during the GC reaction, which may increase antibody affinity for a pathogen. ${ }^{28,29}$ Whether such a mechanism depicted for memory B cells ${ }^{28}$ may also underlie the increased poly-/self-reactivity of the tumor cells of PCNSL, and whether they have been selected for a foreign antigen, is unknown. In this regard, it is of note that the mutation pattern of the IG genes of PCNSL suggested triggering even by a (viral) superantigen., ${ }^{2,3}$

Similar to autoreactive $\mathrm{VH} 4-34^{+} \mathrm{B}$ cells redeemed from elimination in the GC, $72 \%$ of IGHV4-34+ PCNSL showed a mutational loss of the CDR2 sequence motif $f^{4}$ that promotes N-linked glycosylation of CDR2, an event increasing accessibility of the binding site to eliciting foreign antigen. ${ }^{26}$ Thus, one may hypothesize that in PCNSL, naïve precursor B cells may enter the GC where they undergo SHM as part of the rescue process.

However, if the B cells in the process of developing into lymphoma cells or already corresponding to tumor cells were rescued by redemption of autoreactive non-malignant $B$ cells, this process, nevertheless, failed, because it did not yield B cells with reduced auto-/polyreactivity, but rather with increased polyreactivity, although some autospecificities were lost. Interestingly, with progression from the naive to the mutated $B C R$, overall, the $t B C R$ gained autoreactivity for proteins expressed in the CNS including S100 protein family members and constituents of myelin/oligodendrocytes, including MPLZ1, MBP, and MOBP, which are widely and prominently expressed in the CNS, particularly in the white matter and also, at low level, by neurons. ${ }^{30}$ This expression pattern may explain, at least in part, the preferential growth of PCNSL in deep brain structures of the cerebral hemispheres, along fiber tracts, and in the basal ganglia. ${ }^{31}$

Another antigen recognized by $\mathrm{nBCR}$ and/or $\mathrm{tBCR}$ was SNRPC, which is involved in pre-mRNA splicing. ${ }^{32}$ In conditions with chronic B-cell activation, such as systemic lupus erythematosus and other rheumatic diseases, autoantibodies against various ribonucleoproteins are frequent. ${ }^{33-36}$ SNRPC, expressed by the majority of tumor cells in $90 \%$ of PCNSL, may become accessible to tBCR upon necrosis, which is frequent in PCNSL. ${ }^{37}$ These data extend our observation on galectin-3, also expressed on PCNSL tumor cells, ${ }^{10}$ as a potential target for interactions with the BCR. Additionally, $\mathrm{nBCR}$ and $\mathrm{tBCR}$ may recog- nize intrinsic BCR structures, ultimately inducing cellautonomous signaling similar to CLL. ${ }^{38}$

Our data on polyreactive BCR in PCNSL are in line with reports from other mature $B$-cell lymphomas, ${ }^{16,39-41}$ suggesting a role for chronic antigenic stimulation in PCNSL pathogenesis. This hypothesis is supported by a recent study on PCNSL which extended the list of autoantigenic targets by abnormally hyperglycosylated SAMD14 and neurabin-I. ${ }^{42}$ SAMD14 reactivity was shown for 8 of 12 $(67 \%)$ PCNSL with hyperglycosylation of SAMD14/neurabin-I in six $(50 \%) .{ }^{42}$ We noticed a comparable neurabin-I reactivity in 14 of 20 (70\%) PCNSL in an independent series (Online Supplementary Table S1); however, western blots bands were mostly weak (Online Supplementary Figure S9). These data indicate neurabin-I, expressed by neurons and axons (Online Supplementary Figure S10), as potential antigen in a fraction, but not all, PCNSL. Our recAb, however, did not bind to proteins in PCNSL lysates (Online Supplementary Figure S6), because western blot experimental conditions were too stringent. A lack of further concordant data between our work and that of Thurner et al. ${ }^{42}$ may be attributed to technical differences. The ProtoArray and the Unipex differ in their proteins. Focussing on high-affinity antibodies, Thurner et al. ${ }^{42}$ pooled Fab fragments while we designed complete IgG antibodies which were studied individually, considering all recAb-reactive proteins in subsequent analyses.

In conclusion, $B$ cells with auto-/polyreactive $\mathrm{nBCR}$ may enter the GC where SHM may further increase BCR auto-/polyreactivity (Online Supplementary Figure S11). Together with mutation-induced sustained active BCR signaling and the inability to terminate SHM, the cells may become eligible for reaction with a plethora of antigens, particularly of CNS antigens. Thus, in the target organ, a microenvironment fertile for PCNSL proliferation may result from stimulating CNS antigens, autoantigens liberated by dying tumor cells or expressed on the surface of tumor cells, resulting in a vicious cycle of uncontrolled proliferation sustained by the interaction of an active $t B C R$ with (auto)antigens, as a consequence of a faulty GC reaction. While this concept does not fit into the 'classical' view that, physiologically, the GC reaction exclusively selects for high-affinitiy antibodies, it extends novel findings on low-affinity BCR that play a role in immunological memory by adding PCNSL as a lymphoma resulting from a dysregulated GC reaction.

\section{Disclosures \\ No conflicts of interest to disclose.}

\section{Contributions}

$M M R, M T, A B, M D$ and $C M$ performed experiments; $M M R, M T, A B$ and $M D$ analyzed results and prepared the figures; $M M R, A B$ and $M D$ designed the research; $I B$ provided crucial material; IB and KM discussed the manuscript; $M M R$, $R K$ and $M D$ discussed the results and provided important intellectual input; $M D$ wrote the paper; $M H, M M R$ and MT performed statistical analysis.

\section{Acknowledgments}

The authors thank Mariana Carstov, Elena Fischer, and Diana Rudakova for their expert technical assistance.

\section{Funding}

This work was supported by a grant from the Wilhelm SanderStiftung (Grant 2011.092.2) (to $M D$ and $M M R$ ) and the 
Deutsche Krebshilfe (Grant 70112052) (to MD), the Köln Fortune Program (Grant 2680138201) (to MMR), the Medical Faculty at RUB (FoRUM) (CM), the HUPO Brain Proteome
Project (to HBPP) (to CM), PURE and OsteoSys (EFRE 080041) (to CM), de.NBI, (FKZ 031 A 534A) (to CM) as well as Eranet Neuron Silence (FS 4108270133) (to CM).

\section{References}

1. Deckert M, Paulus W, Kluin P, Ferry J. Lymphomas. In: Louis DN, Ohgaki H, Wiestler OD, Cavenee WK, eds. Who classification of tumours of the central nervous system Revised $4^{\text {th }}$ edition ed. Lyon: IARC, 2016:272-277.

2. Montesinos-Rongen M, Küppers R, Schlüter $D$, et al. Primary central nervous system lymphomas are derived from germinal-center $B$ cells and show a preferential usage of the v4-34 gene segment. Am J Pathol. 1999; 155(6):2077-2086.

3. Thompsett AR, Ellison DW, Stevenson FK, Zhu D. V(h) gene sequences from primary central nervous system lymphomas indicate derivation from highly mutated germinal center $B$ cells with ongoing mutational activity. Blood. 1999;94(5):1738-1746.

4. Montesinos-Rongen M, Purschke F, Küppers $\mathrm{R}$, Deckert M. Immunoglobulin repertoire of primary lymphomas of the central nervous system. J Neuropathol Exp Neurol. 2014; 73(12):1116-1125.

5. Serafini B, Rosicarelli B, Magliozzi R, Stigliano E, Aloisi F. Detection of ectopic B-cell follicles with germinal centers in the meninges of patients with secondary progressive multiple sclerosis. Brain Pathol. 2004;14(2):164-174.

6. Roco JA, Mesin L, Binder SC, et al. Classswitch recombination occurs infrequently in germinal centers. Immunity. 2019;51(2):337350.e7.

7. Montesinos-Rongen M, Besleaga R, Heinsohn S, et al. Absence of simian virus 40 DNA sequences in primary central nervous system lymphoma in HIV-negative patients. Virchows Arch. 2004:444(5):436-438.

8. Montesinos-Rongen M, Hans VH, EisHübinger AM, et al. Human herpes virus-8 is not associated with primary central nervous system lymphoma in HIV-negative patients. Acta Neuropathol. 2001; 102(5):489-495.

9. Paulus W, Jellinger K, Hallas C, Ott G, Müller-Hermelink HK. Human herpesvirus6 and epstein-barr virus genome in primary cerebral lymphomas. Neurology. 1993; 43(8):1591-1593.

10. Montesinos-Rongen M, Purschke FG, Brunn A, et al. Primary central nervous system (cns) lymphoma B cell receptors recognize cns proteins. J Immunol. 2015;195(3):13121319

11. Lefranc MP, Giudicelli V, Duroux P, et al. Imgt $(r)$, the international immunogenetics information system(r) 25 years on. Nucleic Acids Res. 2015;43(Database issue):D413422.

12. Hervé M, Xu K, Ng YS, et al. Unmutated and mutated chronic lymphocytic leukemias derive from self-reactive $B$ cell precursors despite expressing different antibody reactivity. J Clin Invest. 2005;115(6):1636-1643.

13. Brändle SM, Obermeier B, Senel M, et al. Distinct oligoclonal band antibodies in mul- tiple sclerosis recognize ubiquitous self-proteins. Proc Natl Acad Sci U S A. 2016; 113(28):7864-7869

14. Hong SH, Cho JG, Yoon KJ, et al. The antibody atliximab attenuates collagen-induced arthritis by neutralizing aimp1, an inflammatory cytokine that enhances osteoclastogenesis. Biomaterials. 2015;44:45-54.

15. Montesinos-Rongen M, Brunn A, Bentink S, et al. Gene expression profiling suggests primary central nervous system lymphomas to be derived from a late germinal center $B$ cell. Leukemia. 2008;22(2):400-405.

16. Sachen KL, Strohman MJ, Singletary J, et al. Self-antigen recognition by follicular lymphoma B-cell receptors. Blood. 2012; 120(20):4182-4190.

17. Wardemann H, Yurasov S, Schaefer A, Young JW, Meffre E, Nussenzweig MC Predominant autoantibody production by early human B cell precursors. Science. 2003; 301(5638):1374-1377.

18. Iype J, Datta $M$, Khadour A, et al. Differences in self-recognition between secreted antibody and membrane-bound $\mathrm{B}$ cell antigen receptor. J Immunol. 2019; 202(5):1417-1427

19. Torres M, May R, Scharff MD, Casadevall A. Variable-region-identical antibodies differing in isotype demonstrate differences in fine specificity and idiotype. J Immunol. 2005;174(4):2132-2142.

20. Tudor D, Yu H, Maupetit J, et al. Isotype modulates epitope specificity, affinity, and antiviral activities of anti-hiv-1 human broadly neutralizing $2 \mathrm{f} 5$ antibody. Proc Natl Acad Sci U S A. 2012;109(31):12680-12685.

21. Spies E, Fichtner M, Müller F, et al. Comment on "primary central nervous system (cns) lymphoma B cell receptors recognize cns proteins". J Immunol. 2015; 195(10):4549-4550

22. Cha SC, Qin H, Kannan S, et al Nonstereotyped lymphoma B cell receptors recognize vimentin as a shared autoantigen. J Immunol. 2013;190(9):4887-4898.

23. Sutton LA, Agathangelidis A, Belessi C, et al. Antigen selection in B-cell lymphomas tracing the evidence. Semin Cancer Biol. 2013;23(6):399-409

24. Montesinos-Rongen M, Schmitz R, Courts $\mathrm{C}$, et al. Absence of immunoglobulin class switch in primary lymphomas of the central nervous system. Am J Pathol. 2005; 166(6):1773-1779

25. Cyster JG, Hartley SB, Goodnow CC. Competition for follicular niches excludes self-reactive cells from the recirculating B-cell repertoire. Nature. 1994; 371(6496):389-395.

26. Burnett DL, Langley DB, Schofield P, et al. Germinal center antibody mutation trajectories are determined by rapid self/foreign discrimination. Science. 2018;360(6385):223 226.

27. Sabouri Z, Schofield P, Horikawa K, et al. Redemption of autoantibodies on anergic $B$ cells by variable-region glycosylation and mutation away from self-reactivity. Proc
Natl Acad Sci U S A. 2014;111(25):E25672575

28. Tiller T, Tsuiji M, Yurasov S, Velinzon $\mathrm{K}$ Nussenzweig MC, Wardemann H Autoreactivity in human igg+ memory B cells. Immunity. 2007;26(2):205-213

29. Diamond B, Scharff MD. Somatic mutation of the 15 heavy chain gives rise to an antibody with autoantibody specificity. Proc Natl Acad Sci U S A. 1984;81(18):5841-5844

30. Seamons A, Perchellet A, Goverman J. Immune tolerance to myelin proteins. Immunol Res. 2003;28(3):201-221

31. Deckert M, Engert A, Brück W, et al Modern concepts in the biology, diagnosis, differential diagnosis and treatment of primary central nervous system lymphoma. Leukemia. 2011;25(12):1797-1807.

32. Brierley CK, Steensma DP. Targeting splicing in the treatment of myelodysplastic syndromes and other myeloid neoplasms. Curr Hematol Malig Rep. 2016;11(6):408-415.

33. Hardin IA, Mimori T. Autoantibodies to ribonucleoproteins. Clin Rheum Dis. 1985; 11(3):485-505.

34. Lerner MR, Steitz JA. Antibodies to small nuclear rnas complexed with proteins are produced by patients with systemic lupus erythematosus. Proc Natl Acad Sci U S A 1979;76(11):5495-5499.

35. McClain MT, Ramsland PA, Kaufman KM, James JA. Anti-sm autoantibodies in systemic lupus target highly basic surface structures of complexed spliceosomal autoantigens. J Immunol. 2002;168(4):2054-2062.

36. Sanz I, Dang H, Takei M, Talal N, Capra JD. Vh sequence of a human anti-sm autoantibody. Evidence that autoantibodies can be unmutated copies of germline genes. J Immunol. 1989;142(3):883-887.

37. Deckert M, Brunn A, Montesinos-Rongen M, Terreni MR, Ponzoni M. Primary lymphoma of the central nervous system--a diagnostic challenge. Hematol Oncol. 2014; 32(2):57-67.

38. Dühren-von Minden $M$, Übelhart $R$, Schneider D, et al. Chronic lymphocytic leukaemia is driven by antigen-independent cell-autonomous signalling. Nature. 2012; 489(7415):309-312.

39. Craig VJ, Arnold I, Gerke C, et al. Gastric malt lymphoma B cells express polyreactive, somatically mutated immunoglobulins. Blood. 2010;115(3):581-591.

40. Seiler T, Woelfle M, Yancopoulos S, et al. Characterization of structurally defined epitopes recognized by monoclonal antibodies produced by chronic lymphocytic leukemia B cells. Blood. 2009:114(17):3615-3624.

41. Zhu D, Bhatt S, Lu X, et al. Chlamydophila psittaci-negative ocular adnexal marginal zone lymphomas express self polyreactive B-cell receptors. Leukemia. 2015;29(7):15871599.

42. Thurner L, Preuss KD, Bewarder M, et al. Hyper-n-glycosylated samd14 and neurabin-i as driver autoantigens of primary central nervous system lymphoma. Blood. 2018;132(26):2744-2753. 